Research Article

\title{
Unlocking the Sustainable Development Path of China's Nonferrous Metal Industry Based on Collaborative Innovation
}

\author{
Jing Zhang $\mathbb{D}^{1,2}$ Yuanyuan Lu $\mathbb{D}^{1,2}$ and Xiangmin Zhang $\mathbb{D}^{1,2}$ \\ ${ }^{1}$ Management School, Henan University of Science and Technology, Luoyang 471023, China \\ ${ }^{2}$ Collaborative Innovation Center of New Nonferrous Metal Materials and Advanced Processing Technology, \\ Luoyang 471023, China \\ Correspondence should be addressed to Xiangmin Zhang; zhangxm@haust.edu.cn
}

Received 11 August 2021; Revised 18 August 2021; Accepted 23 August 2021; Published 15 September 2021

Academic Editor: Daqing Gong

Copyright (c) 2021 Jing Zhang et al. This is an open access article distributed under the Creative Commons Attribution License, which permits unrestricted use, distribution, and reproduction in any medium, provided the original work is properly cited.

\begin{abstract}
The sustainable development of an industry is an important topic of technological innovation. To unlock the path dependence of industrial development, it is critical to identify the evolution law of the industrial collaborative innovation network. Taking China's nonferrous metal industry as an example, this paper establishes two collaborative innovation networks from the time and space dimensions, respectively. The networks cover both the macroenvironment and the microinnovative subjects of the industry and fully consider the collaborative relationships between these subjects. Through multilevel and multidimensional analysis, the authors drew the following conclusions: the macropolicies and technology paradigm can create a window of opportunity for the industry, which directly drives the breeding of new industries and the transformation and upgrading of traditional industries; the interaction between the macroenvironment and microinnovative subjects leads to the differentiation of the industrial innovation path; the provinces can enter the first echelon of interprovincial innovation collaboration and realize sustainable development of the industry through the unlocking path, which consists of path continuation, path expansion, path implantation, and path diversification.
\end{abstract}

\section{Introduction}

The sustainable development of industry is essential to the modern economic system. According to evolutionary economic geographers, path dependence lies at the core of the analysis on regional industry evolution; ideally, the industrial evolution should be a path-dependent mechanism capable of realizing innovation and creating new paths [1]. To pursue a sustainable development of industry, the crux is to unlock the negative locking effect in path dependence. Unlocking the sustainable development path of traditional industries calls for the introduction of new drivers, the key of which is to establish and maintain an effective collaborative innovation network [2].

The collaborative innovation network, involving innovative subjects like enterprises, universities, research institutes, and intermediaries, boasts the advantages of knowledge spillover and technology transfer. The two mechanisms can promote the effective integration and sharing of technological elements [3]. Indeed, innovation, especially collaborative innovation, has been regarded as an effective way for industrial sustainable development.

However, the existing literature on the sustainable development and collaborative innovation provides scant evidence of such mechanisms for industrial high-quality development. For instance, existing studies have explored the influencing factors of collaborative innovation performance from multiple dimensions, such as technology proximity [4-6], geographical proximity $[7,8]$, institutional proximity $[9,10]$, and so on; however, the research conclusions in this respect are very different, and no unified viewpoint has been obtained to guide practice. These static factors cannot effectively reveal the collaborative innovation path and influence the mechanism. Meanwhile, there is little empirical evidence concerning the relationship between the collaborative innovation and sustainable development. In 
fact, understanding the dynamic evolution law of collaborative innovation network is of great value for revealing the growth law of the industry in the course of collaborative innovation and mining the sustainable development path of the industry [11].

To bridge the aforementioned research gaps, our study poses the following question: what is the path of industrial sustainable development in the process of collaborative innovation network evolution? We focus on two evolution dimensions: the time and space, and on this basis, we put forward the path of industrial sustainable development. The study of this problem is helpful to the transformation and upgrading of traditional industries and the breeding of new industries.

The remainder of this paper is structured as follows: Section 2 reviews the relevant literature from time and space dimensions, Section 3 describes the research data and methodology, Sections 4 and 5 present time analysis and spatial analysis, respectively, and Section 6 summarizes the results and provides suggestions to policymakers.

\section{Literature Review}

Industrial development is a process of continuous evolution, transformation, and upgrading of regional industries $[12,13]$. Evolutionary economic geography, which has been developed in recent years, aims to explain the spatial evolution of enterprises, industries, clusters, networks, cities, and regions by analyzing the dynamic processes of enterprise entry, growth, decline, and withdrawal. The historical perspective of evolutionary economic geography can help to link time and space elements and reveal the evolutionary mechanism of the spatial distribution of economic activities [14]. It holds that the development and evolution of regional industries follow the path dependence which are determined by the industrial technology correlation. However, the pathdependent evolution theory lays too much emphasis on the endogenous development process and ignores the pathcreating opportunities brought by exogenous factors and institutional changes. The evolution of regional industry is path dependent, and the economic transformation process of marketization, globalization, and decentralization creates a new path for the development of the regional industry. Behind these, technological correlation plays an important role in creating new paths. Science and technology policy, innovation policy, and industrial policy play an important role in developing new technologies and new industries $[15,16]$. Therefore, evolutionary economic geography believes that the innovation process is rooted in regional institutions. Local institutions and cultures are difficult to be imitated by other regions, and regions become important forces of innovation [17]. The opportunities for the formation of new industries depend on the timeliness and direction of supporting institutions [18]. Institutions cannot be regarded as completely exogenous; they will co-evolve with firm practices.

Meanwhile, research on innovation and sustainability suggests that alliances with external stakeholders help to improve innovation outcomes [19]. Mora-Valentin et al.
[20] believed that the interdependence between the cooperative subjects is the link to construct collaborative innovation, and the higher the dependence between the cooperative subjects, the better the effect of collaborative innovation. Previously, scholars have mainly explored the features of the industrial collaborative innovation network and behavior patterns of the network subjects from the dimensions of time and space. In terms of time dimension, Martin and Sunley [1] found that the video game industry deviates from the typical S-shaped evolutionary trajectory of the industries with strong path dependence because the interfirm network relations and firm survival are moderated of sequences of technological renewal in the industry. Martin and Sunley [1] emphasized that the path breakthrough that enhances external connection promotes industrial development. Mackinnon et al. [21] pointed out that diversified subjects can realize path creation, that is, bringing a new industrial development path.

In terms of spatial dimension, the subject's behaviors of collaborative innovation network have been studied by evolutionary economic geographers and relational economic geographers [22]. From the perspective of relative space, Tanimoto [23] discovered that the industry-university-research collaborative innovation network eventually converges into a scale-free ordered network and explained why human society has been able to develop cooperation even on assortative heterogeneous networks. From the angle of evolutionary economic geography, Mackinnon et al. [21] highlighted the strategic coupling between firms and regions at different spatial scales and revealed that new growth paths emerge out of existing economic activities and their associated assets and conditions. The spatiotemporal evolution of different industries shows different characteristics of innovation networks, but it is concluded that strengthening cooperation is beneficial to break the negative impact of path locking.

The research on collaborative innovation and industrial development has made abundant achievements; however, the research conclusions are different, and no unified point of view has been drawn to guide the practice. The evolution of the industrial collaborative innovation network is influenced by the macropattern and the microbehaviors of innovative subjects [24]. Apart from time and spatial dimensions, it is necessary to reveal the heterogeneity of microinnovation behavioral multiple levels, such as to explore the sustainable development path for a specific industry.

\section{Methodology}

3.1. Methods. Social network analysis is widely used to study the properties, structure, and evolution of social relations to describe and explain the development, change, and innovation mode of society $[25,26]$. The structural features of the collaborative innovation network reflect the collaboration pattern of technological innovation. Through social network analysis, this paper constructs a collaborative innovation network for the collaboration in technological innovation, with each innovative subject as a node and each patent 
collaboration as an edge. The UCINET software $[27,28]$ was used to analyze the overall and individual characteristics of the network, and the evolution characteristics of the innovation network were studied. The evolution characteristics of collaborative innovation network are analyzed with the following network structure indicators.

3.1.1. Structural Hole and Effective Scale. The structural hole represents nonredundant edges in the network [29]. It is a key indicator of the integration of network resources [30]. This indicator can be measured by the effective scale of node $i$, as defined by Burt:

$$
S_{i}=\sum_{j}\left(1-\sum_{k} p_{i k} m_{j k}\right), \quad k \neq i, j,
$$

where $j$ is any node connected to node $i$ in the network, $k$ is any node except $i$ and $j, p_{i k}$ is the probability that node $i$ is connected to node $k$, and $m_{j k}$ is the marginal strength of the edge from $j$ to $k$, that is, the value of the edge from $j$ to $k$ divided by the maximum value among the edges from $j$ to any other node.

The effective scale $S_{i}$ reflects how likely it is for node $i$ to acquire information in the network. Thus, the total effective scale of the collaborative innovation network can be expressed as follows:

$$
S=\sum_{i} \frac{S_{i}^{2}}{\sum_{q} S_{i}}
$$

3.1.2. Intermediate Centrality. Intermediate centrality measures the degree to which a node controls the resources in the network:

$$
C_{B}=\frac{\sum_{i=1}^{n}\left(C_{\mathrm{RB} \max }-C_{\mathrm{RB} i}\right)}{n-1},
$$

where $C_{\mathrm{RB} i}=\left(2 \sum_{i}\left[g_{j k}(i) / g_{j k}\right]\right) /\left(n^{2}-3 n+2\right) \quad\left(g_{j k}\right.$ is the edge between nodes $j$ and $k ; g_{j k}(i)$ is the edge between nodes $j$ and $k$ and passing through $i$ ) and $C_{\mathrm{RB} \max }$ is the maximum value of $C_{\mathrm{RB} i}$.

3.1.3. Average Network Distance. The average network distance $(D)$ refers to the average distance $\left(d_{e_{i}}\right)$ between any two nodes in the network, which measures the strength of the edge between the two nodes:

$$
D=\frac{\operatorname{sum}\left(d_{e_{i j}}\right)}{\operatorname{sum}(E)} .
$$

The smaller the average network distance, the faster the transmission of knowledge, information, and other resources in the network. Therefore, a small $D$ is conducive to the innovation efficiency of the network.

3.1.4. Agglomeration Coefficient. The agglomeration coefficient stands for the degree of clustering among other nodes connected to a node in the network. The philosophy behind this indicator is that two persons are more likely to know each other if they both know the same third person. The agglomeration coefficient $G_{\text {total }}$ can be calculated as follows:

$$
G_{\text {total }}=\frac{3 G_{\triangle}}{3 G_{\triangle}+G_{\wedge}}
$$

where $G_{\triangle}$ is the number of closed three-node groups in the network and $G_{\wedge}$ represents the number of open three-node groups in the network.

3.2. Data. As the output of technological innovation, patent reflects the relationship of collaborative research and development (R\&D), as well as the relationship between the macroenvironment and the microbehaviors of innovative subjects [24]. In this paper, a collaborative innovation network is established for China's nonferrous metal industry based on the data on invention patents applied in the industry, aiming to explore the sustainable development path of the industry.

The patents of the nonferrous metal industry were classified according to the relationship between the International Patent Classification of Nonferrous Metals and the Industrial Classification for National Economic Activities issued by the China National Intellectual Property Administration. Considering the lag between the patent application and approval, the sample period was set as 1985-2017.

Based on the above data acquisition and analysis, the paper analyzed the evolution of industrial collaborative innovation behavior under the influence of macro landscape from the time dimension. Meanwhile, this paper analyzes the industrial development path under the evolution of industrial behavior from the perspective of space and then reveals the path of collaborative innovation to promote the sustainable development of the industry. Figure 1 shows the analysis framework.

\section{Temporal Analysis}

The structural representation of the collaborative innovation network is the basis of evolutionary analysis. First, the social network indices were used to characterize the structural state of the collaborative innovation network involving multiple innovative subjects. After static analysis, the information entropy was introduced to analyze the features and rules of the evolution stage of the collaborative innovation network.

4.1. Network Features. The structural characteristic index of the collaborative innovation network was calculated by formulas (2), (4), and (5) based on the data on collaborative patents in 2000-2017 of China's nonferrous metal industry. Figures 2-5 record the evolution of each index.

Since 2006, the scale of the nonferrous metal collaborative innovation network exploded, with a fast growth of the agglomeration coefficient (Figures 2 and 4). In 2008, the scale of the collaborative innovation network hit a new peak; the network density and agglomeration coefficient both increased rapidly (Figures 3 and 4). However, the industry 


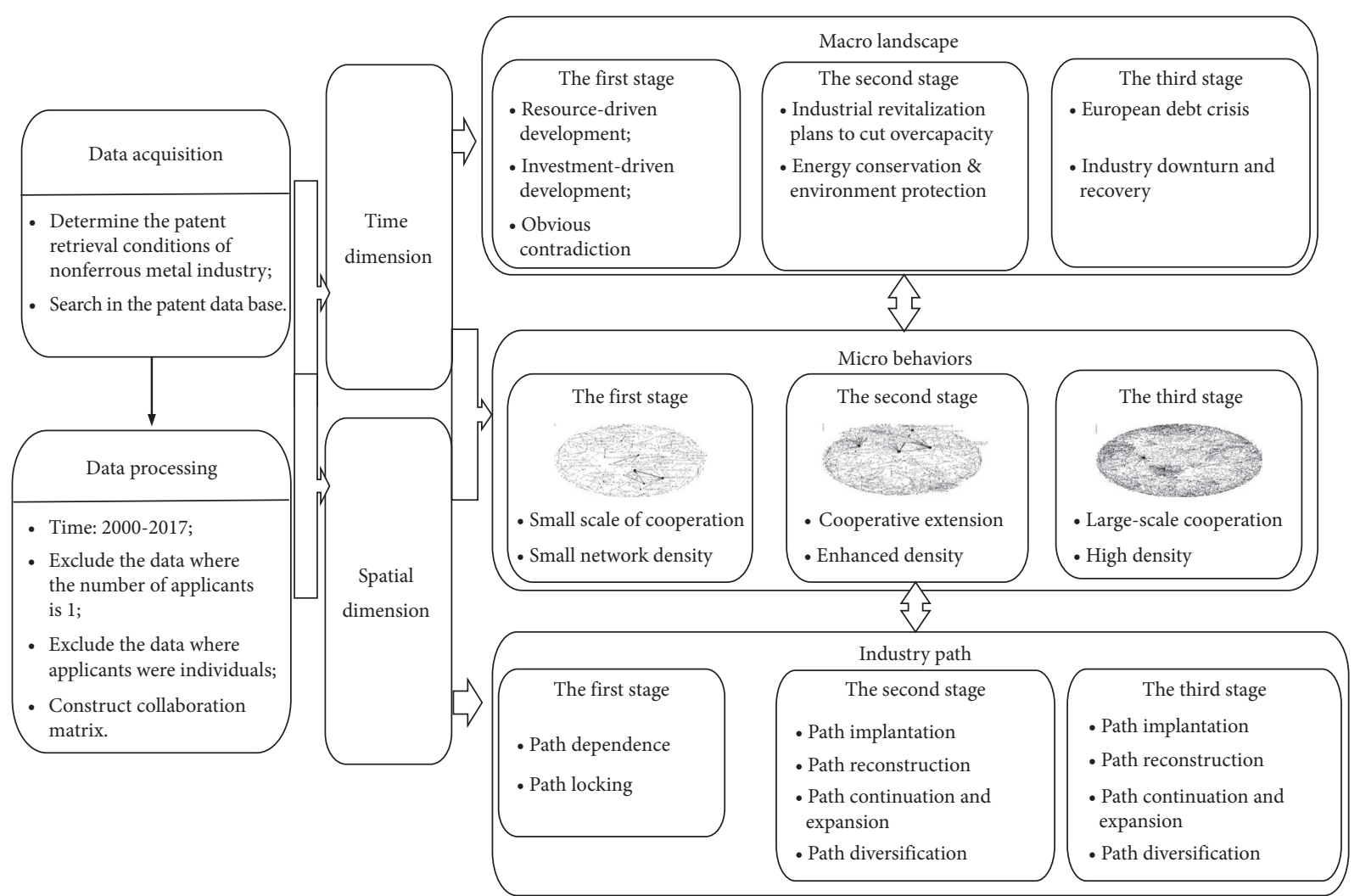

Figure 1: Analysis framework.

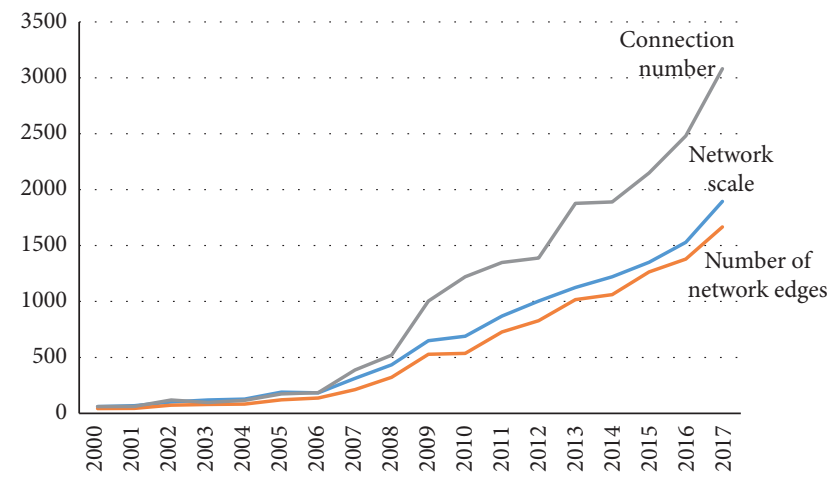

FIgURE 2: Scale evolution of China's nonferrous metal collaborative innovation network.

reached a turning point in 2010; the growth of collaborative patents stalled, and the network became much sparser (Figures 2 and 3). In 2014, the industry entered a period of adjustment, and the number of collaborative patents and the effective scale of the network started to rise again (Figures 2 and 5).

In the two decades, the effective scale of the nonferrous metal collaborative innovation network in China continuously increased, reaching the peak in 2015 (Figure 5). The industry saw a continuous string of innovation agglomerations, and the deepening integration of resources. However, numerical analysis shows that the collaborative innovation network of the industry had a small density and agglomeration coefficient.

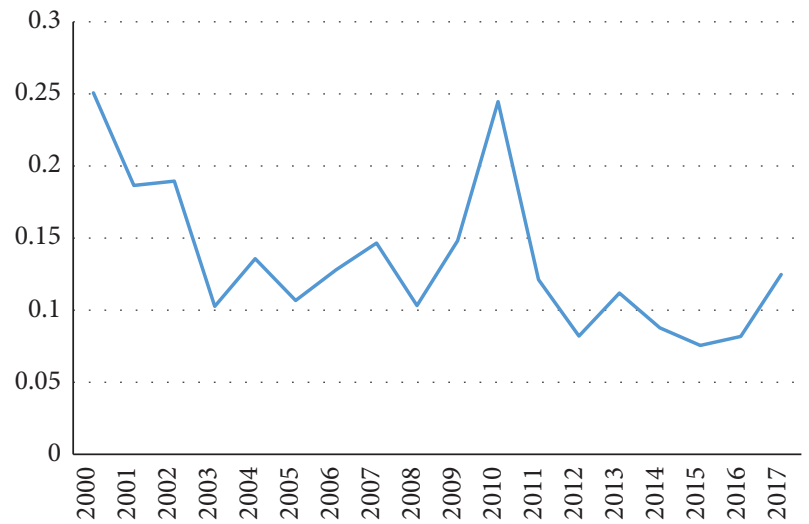

Figure 3: Density evolution of China's nonferrous metal collaborative innovation network.

\subsection{Evolution Stages}

4.2.1. Metric of Evolution Stages. The collaborative innovation network is a complex system, whose structure changes with system orderliness over time. With the previously selected indices of network structure (i.e., effective scale, network density, average distance, and agglomeration coefficient), the overall features of the collaborative innovation network can be described clearly. However, these indices cannot accurately measure the self-organized evolution of network structure. Hence, the concept of entropy was introduced to measure the orderliness of the system [31]. 


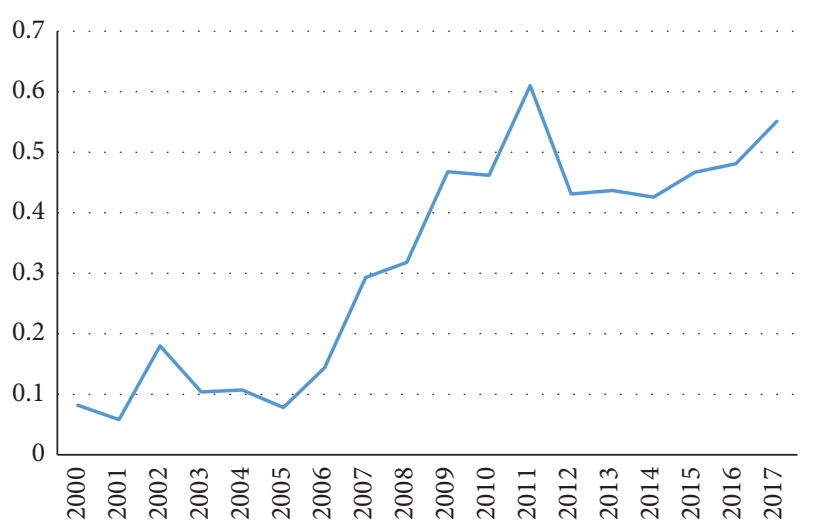

Figure 4: Agglomeration coefficient evolution of China's nonferrous metal collaborative innovation network.

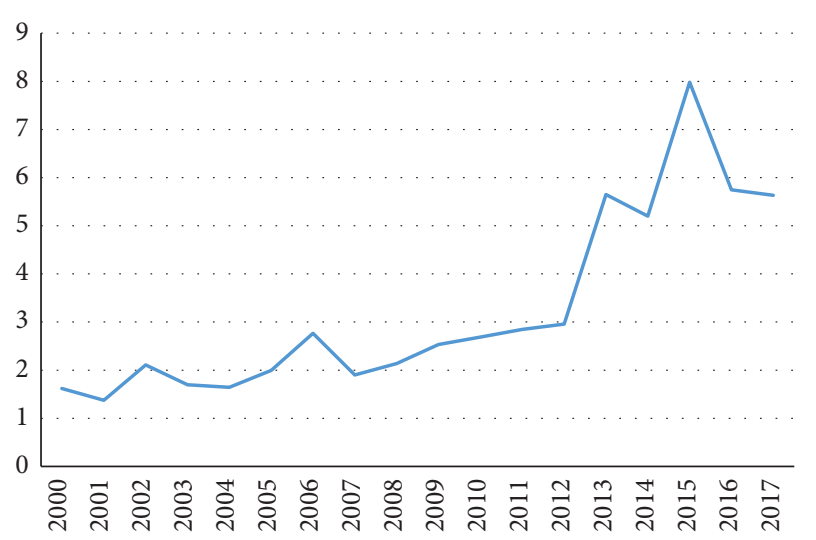

FIGURE 5: Effective scale evolution of China's nonferrous metal collaborative innovation network.

The entropy formula was proposed by Shannon [32], the pioneer of information theory: $H=-\sum p_{i} \log p_{i}$. Obviously, the key to measure the orderly evolution of the collaborative innovation network is to determine the discrete probability of occurrence of events $\left(p_{i}\right)$. Thus, the metric of network structure evolution can be constructed as follows:

$$
\begin{aligned}
& H_{j}=-p_{j} \log p_{j}, \\
& p_{j}=\sum\left(\frac{p_{i j}^{2}}{\sum_{j} p_{i j}}\right),
\end{aligned}
$$

where $p_{i j}=T_{i j} / \sum T_{i j}\left(T_{i j}\right.$ is the periodic observation value of $j$ with the index $i$ ) and $i=1,2$, and 3 is the effective scale $S$, centrality $C$, and agglomeration coefficient $G$ of the network, respectively. Since the average distance $D(i=4)$ is inversely proportional to the orderly evolution of the network, $p_{4 j}=1-T_{4 j} / \sum T_{4 j}$.

4.2.2. Evolution Stage Analysis. The collaboration matrix was imported to UCINET 6.0, and the entropy values in 2000-2017 were calculated using formulas (2)-(6), before being standardized. According to the fluctuation of the standard entropy, the static features of the network, and the macroenvironment, the evolution of the nonferrous metal collaborative innovation network could be roughly divided into three stages (Figure 6):

(i) The first stage (2000-2008): innovation absorption In this stage, the entropy of the collaborative innovation network presented a V-shaped trend. The instability of the trend reflects the imbalanced state of growing entropy. The network scale was smaller than 500, and the agglomeration coefficient averaged at 0.183 . Driven by resources and investment, technological innovation mainly took the form of absorbing technology from abroad. The collaboration was not frequent, and prominent problems existed in industrial development. The network entropy oscillated significantly, and the collaborative innovation network had a loose structure.

(ii) The second stage (2009-2011): transformation and quality improvement

In this stage, the collaborative innovation network witnessed an explosive growth in the agglomeration coefficient, structure hole, and density. The standardized entropy increased from 0.297 to 0.758 . The policy of the Medium and Long Term Scientific and Technological Development Plan for Nonferrous Metals Industry (2006-2020) has promoted the rapid growth of the scale of the collaborative innovation network. In 2009, the Plan for Adjustment and Revitalization of the Nonferrous Metals Industry and the Special Plan for the Utilization of Recyclable Nonferrous Metals were issued, which set the adjustment and upgraded of the industrial structure as the key direction of industrial development, such as eliminating backward production capacity, strengthening technological transformation, and developing recycling and green products. Innovation subjects in the industry, whether vigorously developing deep processing products or energy conservation and emission reduction, pay more attention to the support of technological progress. Outliers in the collaborative innovation network cooperate with other nodes to obtain more innovation resources. Each node in the network enjoyed abundant opportunities to acquire resources, and the network exhibited a trend of expansion.

(iii) The third stage (2012-2017): transitional developments

In this stage, the network edges increased at a much faster rate than innovation nodes. Policies such as the National Medium and Long-Term New Material Talent Development Plan (2010-2020) have encouraged large- and medium-sized key enterprises to establish technology centers and improve technology innovation institutions and talent training. To achieve cost reduction and efficiency increase, on one hand, the industry strengthens the application of energy conservation and emission reduction 


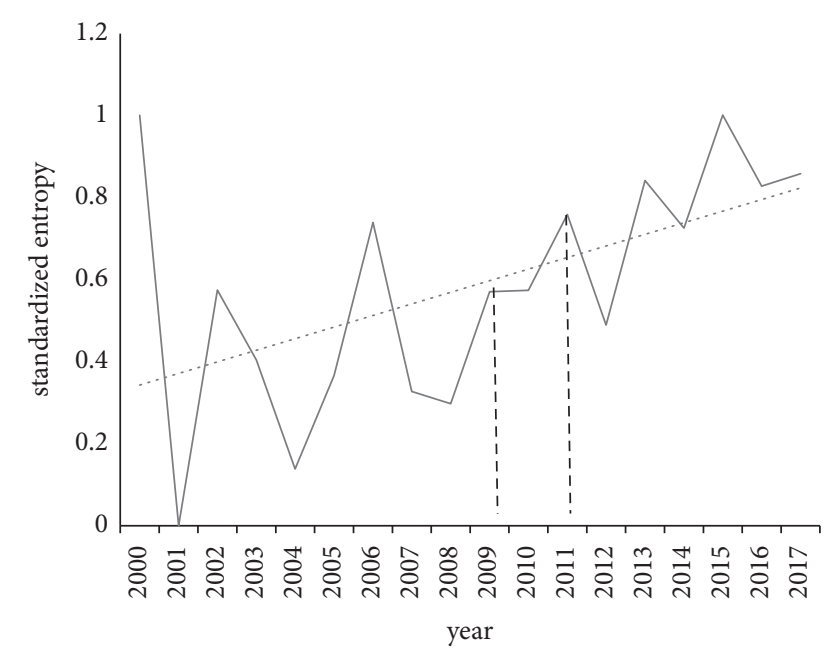

FIGURE 6: Evolution stages of the nonferrous metal collaborative innovation network.

technology, strengthens the control of key processes, and optimizes the production technical indicators. On the other hand, it has established strategic partnership with suppliers, promoted product structure adjustment and technological advantage improvement through investment and acquisition, and opened up foreign markets. The trend of multilateral collaboration was enhanced, giving birth to a knowledge spillover effect (as evidenced by the continued rise of the effective scale). The network entropy followed a chaotic $\mathrm{V}$-shaped trajectory again and continued to increase, owing to the structural adjustment within the industry and the integration with external resources.

From the perspective of time evolution, the nonferrous metal collaborative innovation network evolved from a small network with a few nodes and edges into a large network with many nodes and edges. Through the sample period, the agglomeration degree and resource integration of the network were both improved to a certain extent, but the innovative subjects in the industry were still loosely coupled, that is, the network was not very dense.

\section{Spatial Analysis}

This section attempts to further explore the sustainable development path of China's nonferrous metal industry, in the light of the innovation behaviors of the subjects in the collaborative innovation network. For this purpose, the collaborative patents in the industry were divided by provinces, forming an interprovincial collaborative innovation network.

5.1. Spatial Evolution Features. The patent collaboration in each province could be divided into internal collaboration and external collaboration. All subjects of internal collaboration belong to the same province, while those of external collaboration come from at least two provinces.

The interprovincial collaborative innovation network was constructed based on the internal and external collaborations in various provinces of the nonferrous metal industry, with each province as a node and each interprovincial collaboration as an edge.

Through the sample period, the scale of the interprovincial collaborative innovation network gradually increased, with the number of collaborations growing from 2,146 to 13,122 . The provincial nodes in the network kept gettering closer as the network density grew from 2.3 to 13.2. Overall, the network gradually expanded from the coastal areas in the southeast to the inland areas in the northwest, that is, the nonferrous metal industry slowly shifted to West China during the 12th Five-Year Plan period (Figure 7).

5.2. Evolution Path. To explore the industrial evolution process, a two-dimensional (2D) matrix was established to illustrate the industrial growth in different provinces under different innovation behaviors. In the matrix, the abscissa represents the breadth of collaboration (the network degree), the ordinate represents the depth of collaboration (intensity of collaboration) [33], and the origin represents the mean breadth and depth of collaboration among the provinces. The number of network links and network degree in the three stages are not the same, so the benchmarks of the four categories are not the same across the three stages.

By the 2D matrix, the provinces were classified into four levels: high breadth and high depth ones in the first quadrant (first echelon), high breadth and low depth ones in the fourth quadrant (second echelon), low breadth and high depth ones in the second quadrant (third echelon), and low breadth and low depth ones in the third quadrant (fourth echelon).

To clearly present the data distribution of most provinces, Beijing was not displayed in the matrix (Figure 8) because its collaboration breadth and depth were much higher than those of any other province.

In the first stage (2000-2008), most provinces were concentrated in the second echelon of innovation collaboration, with only Beijing in the first echelon (Figure 8(a)).

In the second stage (2009-2011), the provinces were divided between the first and fourth echelons (Figure 8(b)). Shanghai, Jiangsu, Guangdong, Liaoning, Hebei, Zhejiang, and Henan jumped to the first echelon. This stage is an adjustment period, where China issued policies on overcapacity reduction. The provinces failing to take effective innovative actions fell into the fourth echelon.

In the third stage (2012-2017), the industrial development became more balanced among the provinces as the provinces adapted to the new policies. The main growth path was moving from the fourth echelon to the second echelon and to the first echelon. In this period, Tianjin, Shaanxi, and Shandong jumped to the first echelon.

The three-stage evolution shows the effective qualitative improvement of industrial development path in each province. To find the sustainable development path of the 

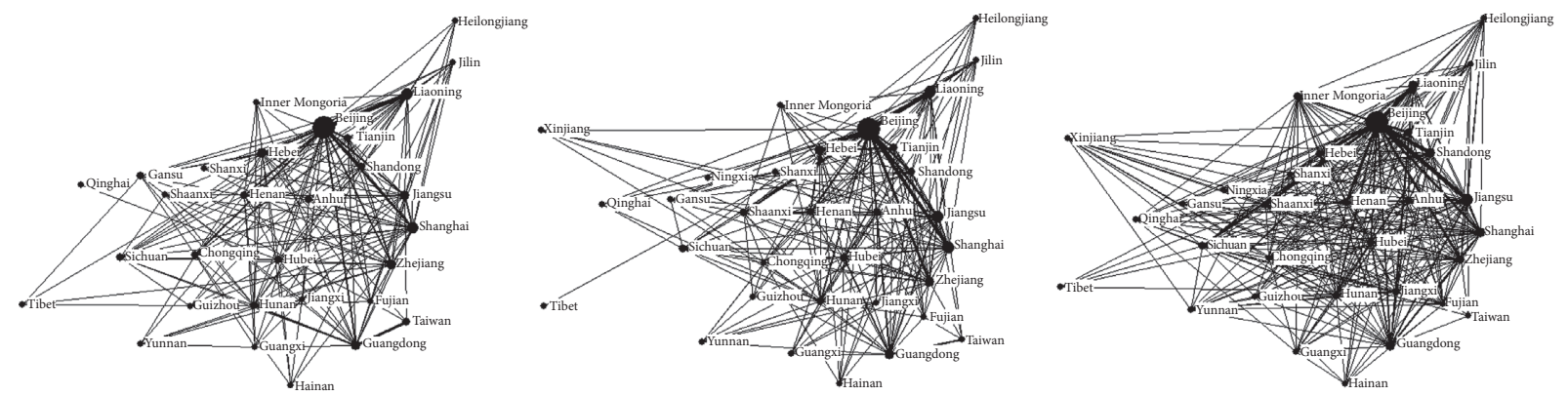

\begin{tabular}{cccc}
\hline Stage & The first stage (2000-2008) & The second stage (2009-2011) & The third stage (2012-2017) \\
\hline Network scale & 31 provinces & 32 provinces & 32 provinces \\
\hline Number of collaborations & 2,146 & 2,668 & 13,122 \\
\hline Network density & 2.3075 & 2.689 & 13.2278 \\
\hline
\end{tabular}

FIGURE 7: Spatial map of interprovincial collaborative innovation network.

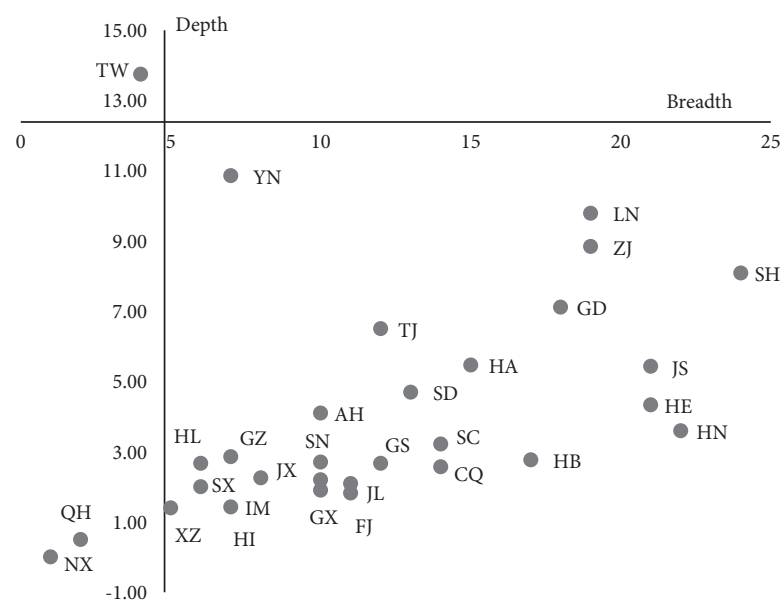

(a)

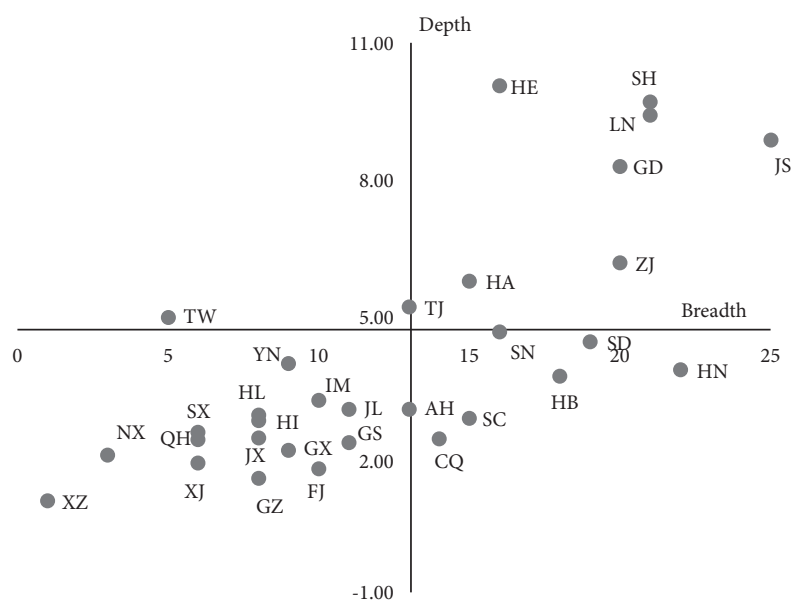

(b)

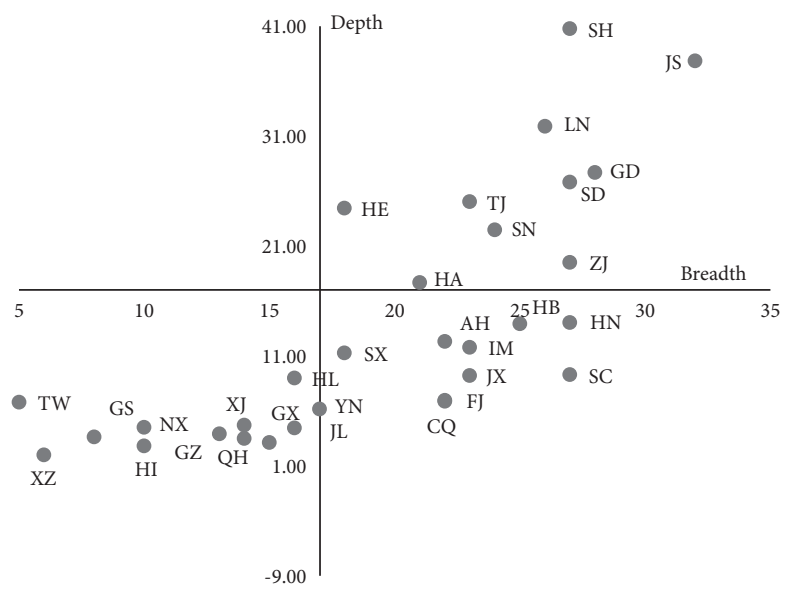

(c)

Figure 8: Breath and depth distributions of interprovincial innovation collaboration. (a) The first stage. (b) The second stage. (c) The third stage. Note: AH: Anhui; BJ: Beijing; FJ: Fujian; GS: Gansu; GD: Guangdong; GX: Guangxi; GZ: Guizhou; HI: Hainan; HE: Hebei; HA: Henan; HL: Heilongjiang; HB: Hubei; HN: Hunan; JL: Jilin; JS: Jiangsu; JX: Jiangxi; LN: Liaoning; IM: Inner Mongolia; NX: Ningxia; QH: Qinghai; SD- Shandong; SX: Shanxi; SN: Shaanxi; SH: Shanghai; SC: Sichuan; TW: Taiwan; TJ: Tianjin; XZ: Tibet; XJ: Xinjiang; YN: Yunnan; ZJ: Zhejiang; CQ: Chongqing. 
industry, it is necessary to focus on two types of nodes in the collaborative innovation network: the provinces leading the industry in all three stages and those making it to the first echelon in the sample period.

5.2.1. Industrial Leaders. Through the sample period, Beijing, Shanghai, Jiangsu, Guangdong, Liaoning, Hebei, and Zhejiang always maintained a high network centrality.

Among them, Beijing and Shanghai realized industrial development by maintaining and enhancing the industrial structure through technological accumulation, as signified by the continued expansion of the innovation path. For example, Beijing and Shanghai formed a high-centrality node early with innovative subjects like Tsinghua University, the University of Science and Technology Beijing, Beijing General Research Institute for Nonferrous Metals, Central Iron and Steel Research Institute (CISRI), State Grid, Baoshan Iron and Steel Co., Ltd., Shanghai Jiao Tong University, Sinopec, and other innovative entities.

Jiangsu and Liaoning evolved into the main innovative subjects in the industry through path continuation, path expansion, path implantation, and path diversification. In the two provinces, research institutions such as Northeastern University, Dalian University of Technology, and Guodian Science and Technology Research Institute became high-centrality innovative subjects in the industry through technological accumulation and path expansion. Meanwhile, the two regions resorted to path implantation and diversification to enhance the sustainability of the industry. For instance, research institutes were brought in to implement path implantation. Many R\&D centers were established by the university and government, namely, Wuxi Research Institute of Applied Technologies, Tsinghua University, and Suzhou Industrial Technology Research Institute of Zhejiang University. In addition, some enterprises expanded the application field of nonferrous metals and diversified the implementation path of brand-new industries. For example, the Jiangsu Huaqiang Electric Power Company upscaled its subsidiary the Jiangsu Huaqiang New Energy Company to handle businesses of nonferrous metals in other application fields.

Guangdong, Hebei, and Zhejiang focused on path implantation by attracting external R\&D institutions and cultivating industrial ecology through talent training. For example, new R\&D institutions, like Yangtze Delta Region Institute of Tsinghua University, were established in Zhejiang. These provinces realized the sustainable development path of the industry by relying on the innovation resources inside the province and achieved a coordinated development of the innovation ecology within the province.

5.2.2. New Entrants. The new entrants to the first echelon mainly include Henan, Tianjin, Shaanxi, and Shandong. Among them, Henan remained a stable proportion of internal and external collaborations through the three stages. From the macrolevel, the industry in this province showed path dependence and lock in of development. From the microlevel, however, the innovative subjects and its partners in Henan always pursued dynamic changes. In fact, the microinnovative subjects in Henan preferred to adopt only one type of development strategy: looking for excellent partners in the industry for collaborative $\mathrm{R} \& \mathrm{D}$. The other sustainable development strategies commonly adopted by other industrial leaders were not implemented in this province. As a result, Henan entered the first echelon in the second stage through collaboration with industrial leaders but was overtaken by latecomers like Tianjin, Shaanxi, and Shandong, which entered the first echelon in the third stage, because of the failure in integrating other development strategies.

\section{Conclusions}

The spatiotemporal evolution of the industrial collaborative innovation network records the history of industrial development. This paper attempts to unlock the path dependence of China's nonferrous metal industry by analyzing the temporal evolution of the macroenvironment of the collaborative innovation network, the spatial distribution of interprovincial innovation collaboration, as well as the behaviors of multiple microinnovative subjects. The main conclusions are as follows:

The temporal evolution of nonferrous metal innovative subjects and the spatial distribution of interprovincial innovation collaboration reflect the heterogeneity of industrial development across China. The heterogeneity explains the law of dynamic changes of the collaboration between microinnovative subjects in the industry. In the second stage, the industry entered the window of opportunity, owing to the macro policies of overcapacity reduction and environmental protection. At the same time, the provinces fell into three echelons by the interprovincial innovation collaboration. The path unlocking strategies for the industry to achieve sustainable development include path implantation, path reconstruction, path continuation and expansion, path diversification, and so on.

To offset the negative effects of market saturation or industrial recession, the only way is to promote the innovative evolution of industrial path and unlock path dependence through continuous innovation. This is the only path toward sustainable competitive advantages [34].

Based on the evolution of China's nonferrous metal collaborative innovation network, several strategies were presented to breed new industries and unlock the path dependence of traditional industries:

(1) To breed new industries, macroplanning (new technology and new market) should be implemented to create a new opportunity space, and knowledge interaction and spillover should be realized within each province through endogenous differentiation and exogenous implantation in the opportunity space, thereby enhancing the technological correlation between industries.

(2) To realize the sustainable development of traditional industries, the provinces with rich innovation resources can unlock the path following path 
continuation $\longrightarrow$ path expansion $\longrightarrow$ path implantation and path diversification/path differentiation and develop into the center of innovative diffusion to drive growth; if the provinces have insufficient innovation resources, the path unlocking procedure could be path implantation $\longrightarrow$ path diversification/ path differentiation $\longrightarrow$ path upgrading.

Specifically, path expansion can be realized through enterprise fission, offshore R\&D institutions, academic entrepreneurship, and so on; path implantation can be carried out through the introduction of $\mathrm{R} \& \mathrm{D}$ institutions and subsidiaries; path differentiation can be achieved by developing new industries related to the old industry based on the development of generic technology; path diversification can be implemented by expanding the field of technology application.

\section{Data Availability}

The data used to support the findings of this study are available from the corresponding author upon request.

\section{Conflicts of Interest}

The authors declare that they have no conflicts of interest.

\section{Acknowledgments}

This research was financially supported by General program of Humanities and Social Science Research, China Ministry of Education, under Grant No. 17YJA630134; Research on Henan Province's Mid- and Long-Term and "Fourteenth Five-Year Plan," Science and Technology Innovation Strategy Layout Program, under Grant No. 202400410004; Soft Scientific Research Projects in Henan Province under Grant No. 192400410094; Higher Education Reform Research and Practice Project of Henan Province under Grant No. 2017SJGLX053; Postgraduate Education Reform Project of Henan Province under Grant No. 2019SJGLX022Y; and Innovation Method Special Work of China Ministry of Science and Technology under Grant No. 2017IM060100.

\section{References}

[1] R. Martin and P. Sunley, "Path dependence and regional economic evolution," Journal of Economic Geography, vol. 6, no. 6 , pp. $395-437,2006$.

[2] R. W. Rycroft and D. E. Kash, The Complexity Challenge: Technological Innovation for the 21st Century, Cassell Academic Publishers, New York, NY, USA, 1999.

[3] W. Fang and L. L. Wang, "Collaborative innovation network: current situation and future trend," Science Research Management, vol. 39, no. 9, pp. 30-41, 2018.

[4] D. C. Mowery, J. E. Oxley, and B. S. Silverman, "Technological overlap and interfirm cooperation: implications for the resource-based view of the firm," Research Policy, vol. 27, no. 5, pp. 507-523, 1998.

[5] A. M. Petruzzelli, "The impact of technological relatedness, prior ties, and geographical distance on university-industry collaborations: a joint-patent analysis," Technovation, vol. 31, no. 7, pp. 309-319, 2011.
[6] R. Narula and G. D. Santangelo, "Location, collocation and R\&D alliances in the European ICT industry," Research Policy, vol. 38, no. 2, pp. 393-403, 2009.

[7] A. B. Jaffe, M. Trajtenberg, and R. Henderson, "Geographic localization of knowledge spillovers as evidenced by patent citations," The Quarterly Journal of Economics, vol. 108, no. 3, pp. 577-598, 1993.

[8] J. S. Katz, "Geographical proximity and scientific collaboration," Scientometrics, vol. 31, no. 1, pp. 31-43, 1994.

[9] P.-A. Balland, "Proximity and the evolution of collaboration networks: evidence from research and development projects within the global navigation satellite system (GNSS) industry," Regional Studies, vol. 46, no. 6, pp. 741-756, 2012.

[10] H. Wei and S. Y. Sung, "The effect of institutional proximity in non-local university-industry collaborations-an analysis based on Chinese patent data," Research Policy, vol. 42, no. 2, pp. 454-464, 2013.

[11] C. Freeman, "Networks of innovators: a synthesis of research issues," Research Policy, vol. 20, no. 5, pp. 499-514, 1991.

[12] M. Ron, "Roepke lecture in economic geography: rethinking regional path dependence: beyond lock- in to evolution," Economic Geography, vol. 86, no. 1, pp. 1-27, 2010.

[13] M. Ron and S. Peter, "Path dependence and regional economic evolution," Journal of Economic Geography, vol. 6, no. 4, pp. 395-437, 2006.

[14] M. Storper, "The limits to globalization: technology districts and international trade," Economic Geography, vol. 68, no. 1, pp. 60-93, 1992.

[15] C. Criscuolo, R. Martin, and H. G. Overman, "Some causal effects of an industrial policy," American Economic Review, vol. 109, no. 1, pp. 48-85, 2019.

[16] M. Mazzucato, "Mission-oriented innovation policies: challenges and opportunities," Industrial and Corporate Change, vol. 27, no. 5, pp. 803-815, 2018.

[17] W. M. Feng and X. H. Xue, "The evolutionary paths of new emerging industry in cities: a case study of the Internet of things industry in the Yangtze River Delta region," Geographical Research, vol. 34, no. 9, pp. 1697-1707, 2015.

[18] R. R. Nelson, "Co-evolution of industry structure, technology and supporting institutions, and the making of comparative advantage," International Journal of the Economics of Business, vol. 2, no. 2, pp. 171-184, 1995.

[19] E. A. Inigo, P. Ritala, and L. Albareda, "Networking for sustainability: alliance capabilities and sustainability oriented innovation," Industrial Marketing Management, vol. 89, pp. 550-565, 2020.

[20] E. M. Mora-Valentin, A. M. Sanchez, and L. A. Guerras-Martin, "Determining factors in the success of R\&D cooperative agreements between firms and research organizations," Research Policy, vol. 33, no. 1, pp. 17-40, 2003.

[21] D. Mackinnon, S. Dawley, M. Steen et al., "Path creation, global production networks and regional development: a comparative international analysis of the offshore wind sector," Progress in Planning, vol. 130, no. 5, pp. 1-32, 2018.

[22] A. L. Barabási and R. Albert, "Emergence of scaling in random networks,” Science, vol. 286, no. 5439, pp. 509-512, 1999.

[23] J. Tanimoto, "Coevolutionary, coexisting learning and teaching agents model for prisoner's dilemma games enhancing cooperation with assortative heterogeneous networks," Physica A: Statistical Mechanics and Its Applications, vol. 392, no. 13, pp. 2955-2964, 2013.

[24] H. Hakansson, "Industrial technological development: a network approach," International Journal of Research in Marketing, vol. 4, no. 2, pp. 157-159, 1987. 
[25] R. D. Alba and C. Kadushin, "The intersection of social circle: a new measurement of social proximity in network," Sociological Methods and Research, vol. 5, no. 1, pp. 77-102, 1976.

[26] S. P. Borgatti and P. C. Foster, "The network paradigm in organizational research: a review and typology," Journal of Management, vol. 29, no. 6, pp. 991-1013, 2003.

[27] J. D. Johnson, "UCINET: a software tool for network analysis," Communication Education, vol. 36, no. 1, pp. 37-41, 1987.

[28] A. D. James, "Identifying collaborative innovation networks at the inter-departmental level," Procedia Social and Behavioral Sciences, vol. 2, pp. 6304-6417, 2010.

[29] L. Leydesdorff and I. Rafols, "Local emergence and global diffusion of research technologies: an exploration of patterns of network formation," Journal of the American Society for Information Science and Technology, vol. 62, no. 5, pp. 846860, 2011.

[30] R. S. Burt, Structure Holes: The Social of Competition, Harvard University Press, Boston, MA, USA, 1992.

[31] B. Li and X. Zhu, "Research of RIS' evolutionary mechanisms based on the theories of dissipative structure \& grey relation entropy," Studies in Science of Science, vol. 25, no. 6, pp. 1239-1243, 2007.

[32] C. E. Shannon, "A mathematical theory of communication," The Bell System Technical Journal, vol. 27, no. 4, pp. 379-423, 1948.

[33] J. F. Yuan and Z. Xu, "Research on the structural characteristics and evolution of industry-university-research cooperation networks in China: based on analysis of patent data from 1985-2013 years," Chinese Journal of Management, vol. 14, no. 7, pp. 1024-1032, 2017.

[34] S. Zhu, C. He, and Y. Zhou, "How to jump further and catch up? path-breaking in an uneven industry space," Journal of Economic Geography, vol. 17, no. 1, pp. 521-545, 2017. 